\title{
$\begin{array}{llllllll}\text { E } & \mathbf{P} & \text { I } & \mathrm{S} & \mathrm{T} & \mathbf{O} & \mathbf{L} & \mathbf{A}\end{array}$
}

\section{$\Delta \mathrm{D}$ \\ FRIDERICUM JACOBSIUM}

\author{
VIR UM CLARISSIMUM
}

DE ANTHOLOGIA GRAECA ET LITTERIS OMNINO

GRAECIS IMMORTALITER MERITUM. 



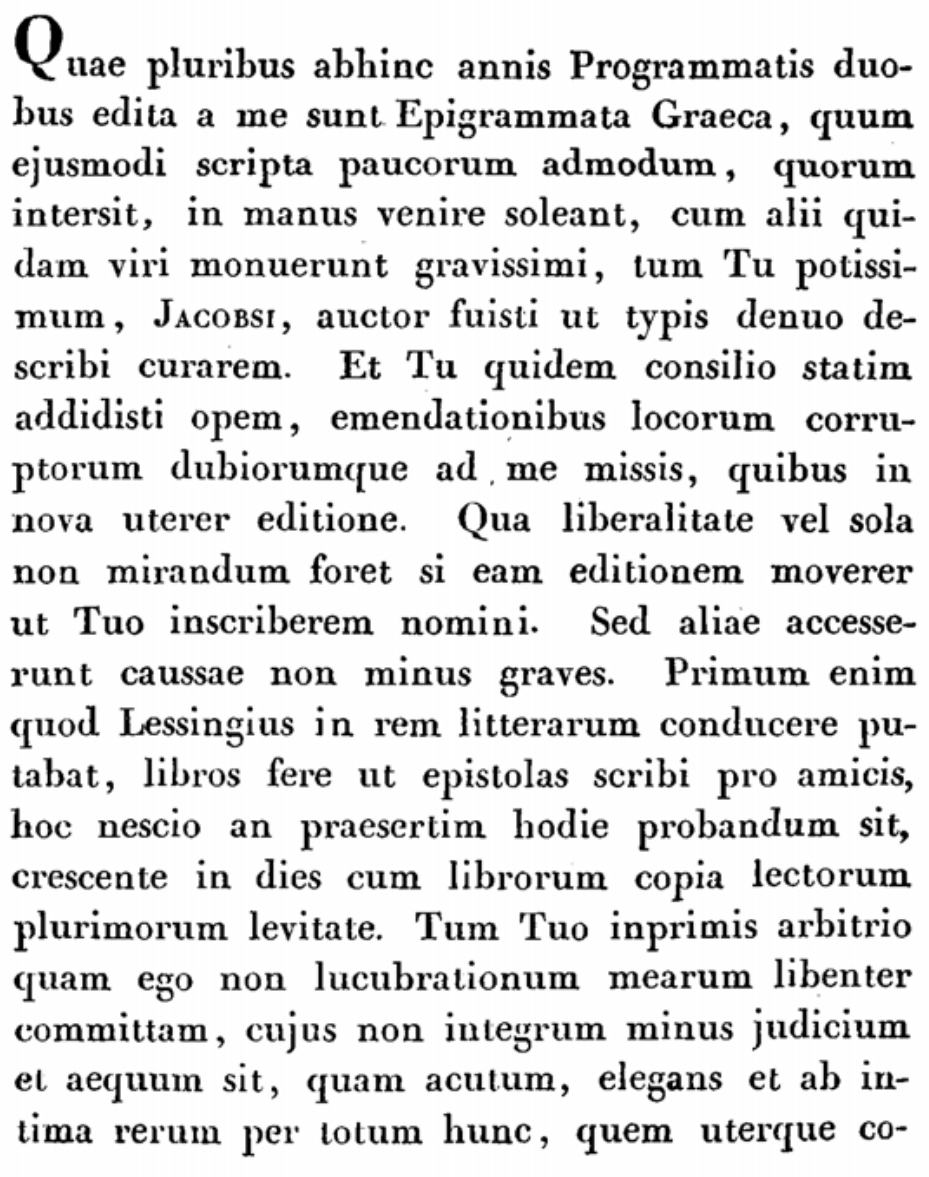


limus, campum, cognitione instructum? Et epigrammata quidem Graeca tractans, quisquis sit, ad quem prius dirigat animum, quam ad Te, quo neminem huic generi post renatas litteras intentiorem et diuturniorem adhibuisse curam, operam magis conducibilem et fructuosam, inter omnes constet? Me vero hoc quidquid est epigraphici operis ad te afferre tanquam appendiculam ad largam Tuam Epigrammatum Appendicem applicandam, Tibi dicare et in Tuo quasi nomine edere eo etiam par est, quod Tuo exemplo, quum in ultimo Anthologiae Palatinae volumine occupatum Te ex litteris cognovissem, primum excitatus sum, ut dispersa epigrammata circumspicerem et indagarem. Quamvis enim inscriptiones veteres ubicumque occasio ipsa marmora videndi data fuisset, quorum adspectus rerum exhibitarum momenta mirifice augere solet, cupide consideraveram, neque editas, his semel deliciis captus, prorsus neglexeram, at egeram hoc tamen quasi in transcursu et magis animi causa quam seria opera et continua. Tum vero et libris multis evolutis et schedis excussis, quae nondum a Te occupata videbam, misi epigrammata, quae Tu Addendis inseruisti. Postea nova identidem accesserunt, et quum a. 1819 forte ac subito negotium imponeretur, ut prima, quae Rhenana Academia celebravit, sollemnia academico more indicerem, promsi quantum aderat in pub- 
licos usus. Quo fecerim conșilio, qua ratione, praesertim quum non mutaverim, iisdem, quibus olim, verbis declaro. Dixi igitur, quod Tu, Jacobsi, Brunckii secutus exemplum, epigrammata ex libris et marmoribus collecta utrisque Tuis, quibus aeternum de Anthologia Graeca promeritus sis, editionibus, corollarii loco, subjunxeris, gratiam habere et semper habituros esse omnes Graecarum litterarum studiosos, tum quia multa sint inter ea sensuum atque dictionis nativa venustate et grata simplicitate insignia, quae in libris minus vulgaribus, non ad litterarum Graecarum, sed ad reconditiorem antiquitatis scientiam pertinentibus delitentia, multorum poeseos Graecae condorum promorum vel ex doctissimis notitiam fugere potuissent, tum quia variis hujus generis reliquiis in unum collatis et cumulatis facilius, quam late patuerit inter Graecos sermonis poetici et metrorum peritia quaedam et ufus, quatenus scilicet ex lapidariae Musae fetibus hoc aestimetur, censeri atque computari possit: Quare operae pretium visum esse, si quae epigrammata vel dudum edita a Te et Brunckio praetermissa, vel nuperrime in itinerariis vel ubiubi in lucem protracta reperissem, ea ut in fasciculos subinde constricta ad largas messes Vestras apponerem; non quo curiose omnia excuterem, docteque et copiose illustrando absolverem, sed ne nimium quantum dispersa diutius a multis ignorari paterer. $\mathrm{Ne}$ - 
que soli Anthologiae editores, ipsorum ejus conditorum hac in re similes, epigrammata ex marmoribus vel libris epigraphicis conquisivere, sed fuerunt et alii, qui de carminibus ex inscriptionum farragine seligendis et ad poeseos thesauros transferendis cogitarunt. Scipioni Maffejo, egregio viro, in universali, quod parabat, Latinarum veterum et Graecarum inscriptionum Corpore epigrammata versibus concepta, a reliquis sejuncta, in poesis gratiam, ut ipsius utar verbis in Sigl. lapid. p. 128 . 132 , in unum colligere et consociare propositum fuit: quo motum suspicor Bonadam, hominem nec ingenio nec litteris instructum, ut ederet $A$ nthologiam sive collectionem omnium veterum inscriptionum poeticarum tam Graecarum quam Latinarum in antiquis iapidibus sculptarum in decem classes distributam 1751. Mihi collectio rectius in tres classes distribui posse videtur, titulos $\grave{\alpha} \nu \alpha-$ $\Im \eta \mu \alpha \tau \iota x o v ' s$, qui statuis, aris, donariis insculpti fuerunt, sepulcrales et mistos, ita quidem, ut classes in capita quaedam dividantur, prima igitur ut secundum certum personarum et rerum, deorum et heroum, magistratuum et privatorum, civitatum etiam ordinem, sepulcrales ut in publicos et privatos dispertiantur, et illa quidem ad civitatis, haec ad domus et familiae et totius vi'tae humanae imaginem quandam rite adumbratam commode ac solerter disponantur, temporis simul ubique, quantum fieri liceat, ratione ha- 
bila. Tertia, quam dixi, classis reliquos omnes titulos carmine decurrentes continebit, numero pauciores, quorum varius fuit usus, quum Graeci etiam significationes variarum rerum et indicia, nuntios, monita, denunciationes, sententias, dicteria versus aliquo ornatu instructa in locis publicis inscribere soliti sint. In ejusmodi autem opere conficiendo facile apparebit epigrammata etiam omnia, quae ab antiquis ex marmore et aere descripta vel testium fide vel argumento ipso constet, simul ad censum vocari et magno cum fructu ad utrorumque intelligentiam et ad facilius pleniusque ex cunctis vel rem antiquariam, vel poetarum morem, consensum et varietatem cognoscendam ex Anthologiae sectione sexta et septima, ubi divęrsae indolis plurima is immista sunt, et ex reliçuis, quo istius generis quaedam aberrarunt, traducta, suo quodque loco inseri posse. Dorvillium quaenam ad novam Epigrammatum tum ex saxis tum ex codicibus editionem praeparantem mors occupaverit, quod factum est duo annos post Bonadae editum librum, Burmannus in praefatione Anthologiae Latinae p. VII refert. Viscontius, Astorgio narrante in Epistola in Anthol. Bosch. Vol. V p. XXXVII, fuit quum ineditorum Epigrammatum syllogen e lapidibus concinnare in mente haberet, quam Hieronymo de Bosch edendam traditurus erat.

In altera hac editione ut quinam ex priore 
repetiti tituli sint in oculos incurreret, eorum numeros novis, qui nunc impositi sunt, uncinis inclusos adjeci. Feci etiam ideo, quod illis pariim contigit, ut animadversionibus meis suas viri quidam eruditissimi adderent. Nam non diu post quam specimen prius edideram a. 18!9, Fridericus Graefe, Vir Graece doctissimus et de epigrammatica poesi jam antehac egregie meritus, simili instituto, Inscriptiones Graecas ex antiquis monumentis et libris editis depromptas restituit et explicavit in Dissertationibus duabus lectis in consessibus Academiae Petropolitanae mense Martio et Octobre a. 1821 et mox impressis in Actis Academiae T. VIII, 1822 p. $629-708$. In quo opere plura epigrammata in illo specimine exhibita denuo tractavit et pluribus locis felicissime restituit, quae nunc leguntur n. 11. 36.39.52. 74; simulque alia, quae a me in altero fasciculo eodem anno 1822 edito, antequam vel Graefii commentatio in meas manus venerat vel alter libellus meus ad Graefium allatus fuerat, edita sunt, n. 6. 28. 32. 36. Utroque autem programmate perlecto Niebuhrius, quo est quorumcunque utriusque linguae monumentorum juvandi studium prompto animo, observationum suarum copiam ultro mihi fecit humanissime, quem erga opellam meam favorem et ipse libentissime acceptum retuli, et multi litteratorum hominum, qui rebus faveant, de quibus agitur, praedicabunt. Bockkhio, çuan- 
tum tota haec Sylloge debeat, quaevis fere pagina loquitur: qui non in excusis tantum ad emendationem et explicationem plurimum contribuerit, sed per litteras etiam et lectiones et tota epigrammata quaedam mecum communicaverit. Idem, pro amicitia sua, quum jam inibi esset, ut liber typographo traderetur, tertii fasciculi majorem partem antequam absolutus et vulgatus esset ad me misit, unde factum est, ut non nisi ultima folia, inde a p. 820 , post impressum jam librum meum acciperem, ex quibus quae ad illum spectant in Addendis excerpsi. Interim etiam Osannus in ampla, quam edere continuat, Sylloge Inscriptionum, plures, quas in hanc nostram recepi, suis annotationibus instruxit; qui quidem Vir doctissimus etiam inedita quaedam milhi tradidit. Eandem debeo gratiam Eduardo Gerhardo meo, Viro optimo, qui nunc denuo in Italiam reversus ad vasa picta aliaque artis opera edenda praeclaro studio totum se applicat, et Letronnio, qui accurata doctrina et eximio acumine Galliam litteratam ornat et illustrat. Ab hoc enim duo accepi epigrammata non antea prodita; ille autem plura mihi olim Romae ex marmoribus, quae ultimis hisce annis terra eruta sunt et partim in Diariis Romanis edita, multa impensa opera, accurate transcripsit. Subsidiis his luculentum accessit et gratissimum augmentum, Tuarum annotationum, amicissime $\mathrm{J}_{\mathrm{ACOBSI}}$, nova et copiosa symbola. Non 
speraveram equidem quum Syllogen antequam imprimeretur ad Te mitterem, ut librum Tibi prae aliis scriptum prius etiam quam alii videres et vel legere vel percurrere posses, neque a Te expetiveram, quem ab omnibus Musis per vices et certatim invisi et post absolutas Lectiones illas Stobenses, ingeniosarum et exquisitarum correctionum et conjecturarum plenum Amaltheum, Aeliano otium addixisse scirem, ut illum critica diligentia nunc quidem pertractare et annotatis Tuis augere et ornare velles. Tu vero tota sine mora farragine examinata, tantam notarum et emendationum copiam mihi obtulisti, quas Tuis plerumque verbis locis suis adjeci et inserui, ut, post Philostrateas nostras Imagines, hunc etiam librum communi nostra opera atque cura confectum in vulgus exire possim gloriari.

Magna est et multiplex in his epigrammatis diversitas, nec rerum et argumenti major quam ingenii atque cultus: quae tot seculorum spatio partim in urbibus Graecis a Graecis, partim a Romanis in terris peregrinis, a poetis alia et poeseos studiosis, alia ab indoctis hominibus composita sint. Et plurima inter ea, si singula per se spectes, minimi esse pretii, nemo me vel melius intelligat vel libentius agnoscat. Ilque eo minus mirandum, quod maxima pars ab hominum privatorum vel cippis et sarcophagis vel statuis, quibus pro lemmatis fuerunt, desumta est. Quanta 
tamen Graecorum in hoc etiam poeseos genere praestantia fuerit, facile discet cui comparationem instituere, et, quum imaginibus titulos adscribi minus etiam in usu hodie sit, coemeteria perlustrare libuerit. Quo facto redeat mihi ad Anthologiae librum septimum, ad illud quod in Appendice ex disjectis lapidibus et ut fors ferebat vario tempore ex terra effossis constructum est $\pi 0 \lambda v \alpha \dot{\nu} \delta \rho \iota \nu$, et ad hoc etiam nostrum: et scio, magis, quam antea eum esse admiraturum simplicitatem istam cum elegantia et varietate in rebus vulgaribus enarrandis conjunctam; et in pluribus etiam humanitatem in communi generis nostri sorte et intime sentienda et aeque ferenda. Multae sunt inter sepulcrales hujus Sylloges jambico metro, quod ad simplicem narrationem magis, quam ad epicedium aptum est, conscriptae, n. 19 -21. 23. 32. 59. 79. 81. 89.113.115, choliambico n. 49 et 80 ; nec multo major numerus earum est, quae in Anthologia et in Appendice eodem conceptae versu servatae sunt non $\dot{\varepsilon} \tau \iota \delta \varepsilon \iota \kappa \tau \iota x \alpha i$, sed cippis revera olim adhibitae, in illa quidem Epitymb. n. 155. 281. 293, quae in eodem cippo, quod Tu quoque statuisti, Jacobsi, cnm elegiaco epitaphio, quod praecedit, insculpta fuit, ut apud nos n. 22. 23 et n. 8 1. 82 ; porro n. 59 4. 695 . Quibus accedunt ex Appendice n. 57. 20/4. 256. 331.343. Apollonidae jambi in piscatorem Epitymb. n. 693 ficticium sunt epitaphium, sicut et ii, quos An- 
tipater n. 692 et Philippus Planud. IV, 52 in athletas victoriarum numero supcrbientes, multo sale conditos et agonisticos titulos imitati conscripserunt, et Hipponactei in Philaenidem Epitymb. n. 345. Senariolos quosdam in Archimedis motutmento inscriptos, partim contritos et obscuratos Cicero memorat Tuscul. V, 23. Anthologia Latina in Libro Epitaphiorum quindecim continet trimetris concepta, inter quae sunt admirabile illud in Fabiam Paulinam n. 201 et elegantes senarii in Mimam Liciniam, de quibus $v$. Viscontius in Iconogr. Graeca tab. 37. Addas his Pacuvii jambos ap. Gell.. I, 24 et inscriptum Dosseni monumento titulum ex Senec. Epist. 89. Etiam Christiani senariis Graecis in cippis haud raro usi sunt. V. fragmentum Mus. Veron. p. 363, 3. Gruter. p. 420, 7. 1048. 2. Ex trochaicis tetrametris constat Atticum epitaphium in rhetorem Harpocralionem Append. n. 320 (Boeckh. n. 923.)

Quaedam nunc omisi, quae in ordinem an tea receperam, duas sepulcrales inscriptiones Romanas, postremum a Vermigliolio editas in Inseriptionibus Perusinis p. /,3o, fragmentum, quod Boeckhius dedit n. 11/47, haud ineptum id fortasse, sed cujus correctius exspecto apographum,

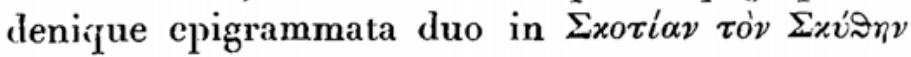
et Timonem Misanthropum in Obsidione Corinthi cadentem, quae ex Diarii Classici Vol. XVI p. 395, quamquam "portentosa" erant visa, tamen exscri- 
pseram. Quam non sint portentosa, sed lepida, docuit me Niebuhrius, et vidi accidisse mihi, quod plures saepe experiri videntur, ut in is, quae Graece scripta legant vel Latine, quasi antiquitatis et antiquarum litterarum ac phrasium quodam fascino constricti, acumina, argutias, jocos, sensus non perspiciant, quae vel mediocriter doctus et perspicax, si in libro novicio in ea incideret, nullo negotio perciperet.

Ceterum multum abfui a Brunckii rationibus (Lectt. et Emendd. p. 3or) et morositate, cui non nisi ea, quae boni commatis essent, Anthologiae addere visum est (quasi boni commatis essent omnia, quae Anthologia continet): nam saxea in saxis legi satis esse. Quare ex solo Veronensi Museo, unde Brunckius novem deprompserat epigrammata, Tu duo alia adjecisti (Append. n. 315 et Not. crit. p. XCIX, quamvis hoc illic ex Monumentis Gabinis profers), sex alia sumsi, n. 19. 56 . 72. 76.83 et 86 ; in Gruteriano autem Thesauro undecim mihi relicta erant, n. 4o. 41.42. 49. 73 . 76. 77. 80. 84. 91. 165, postquam Brunckius inde hauserat viginti octo, quibus accesserunt in Appendice Tua n. 137. 153. 155. 160. 289. Ex Muratorii collectione affero n. 59.61.65.81.87.90; quaedam etiam apud Reinesium, Gudium, Hesselium inveni non praecepta, n. 78. 100. 138. Exclusi autem nihil omnino eorum, quae vel in lapidibus inventa metro adstricta sunt, vel in li- 


\section{- $\mathrm{xvr}-$}

bris relicta erant ad genus Epigrammatum aliquo modo pertinentia; praeter titulos nimis truncos atgue laceros, quorum plurimi sepulcrales sunt, satis partim longi, ut quidam in Corp. Inscr. et is, qui in Diario Arcadico 1825 mense Decembr. editus est in hominem Aegyptium Romae mortuum, et praeter eos sepulcralium, qui membra potius versuum quam versus continent, ut Perusini duo, quorum mentionem feci, et ii, qui in Corp. Inscr. n. 1655, in Anim. ad Anthol. T. III P. 2 p. $31_{7}$ et in Append. n. 252 editi sunt. Ejusdem generis sunt Latina ex Grutero citata a Burmanno ad Anthol. Lat. IV, 137, quos ille mira quadam superstitione, ex qua malos versus a Graecis et Romanis procudi potuisse credere nefas nonnullis videtur, non aliam ob caussam tam rudes esse opinatur, quam quia superstites luctum exprimere voluerint "adeo ipsos externantem, ut qui sint, ubi sint quidve loquantur non sibi nosse viderentur." Neque tamen misti hujus generis prorsus expers est Sylloge nostra (v. n. 41 et 90); et addere ex eodem libet hoc loco inscriptionem a Sponio olim in Miscellan. p. 348 editam, quia pluribus premitur difficultatibus, quarum unam alteramve removere conabor. Insculpta illa est cippo quadrilatero grandi, in hortis Borghesianis exposito, qui Lutetiam non videtur transportatus esse, et a Sponio, cujus apographum cum alio. a Georgio Zoega facto (in schedis ad anaglypha per- 
tinentibus p. 487) contuli, accurate transcripta, nisi quod litteras $\epsilon c \omega \mu$ vulgaribus formis expressit. Habet etiam Fleetwoodius p. 85 n. 1, qui quid illa sibi velit se non intelligere fatetur, ut fere semper nihil intelligit. Quem titulum in commata sua vide an recte ita disposuerim:

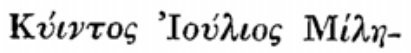

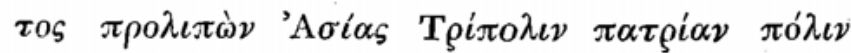
$\dot{\alpha} \gamma \boldsymbol{v} \boldsymbol{\eta}^{\nu}$,

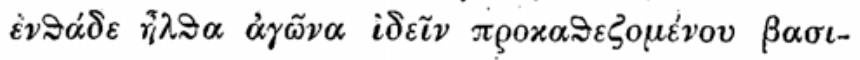

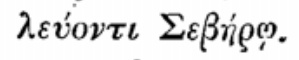

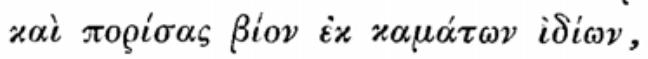

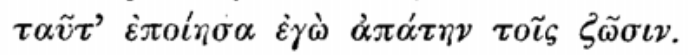



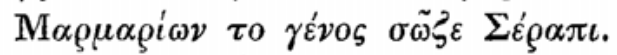

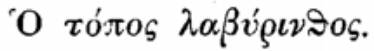

V. I legitur חATPIAAN. Mirus est error constructionis in $\beta \alpha \sigma \iota \lambda \varepsilon v ́ o v \tau \iota$, fere ut in epigrammate modo citato App. n. 252 T⿳亠े冖 $-\lambda \alpha \beta o \tilde{v} \sigma \alpha \nu-\dot{\alpha} \lambda \lambda^{\prime}$

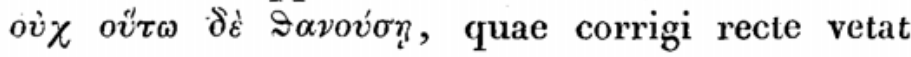
Boissonadius Epist. Holsten. p. 432 . V. 3 غे $\approx \alpha \mu \alpha$ $\tau \omega \nu$ i $i^{\prime} \omega \nu$, ut solent qui magnas adquisiverunt opes, peregrina negotia vel lucrosas provinciarum

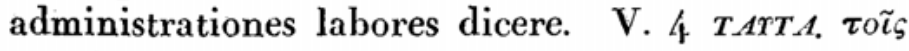
$\zeta \tilde{\omega \sigma \iota \nu, ~ q u i a ~ m o r t u u s ~ l o q u i t u r . ~ V . ~ 5 . ~ r e s t i t u i ~} \varepsilon \dot{\varepsilon}$ -

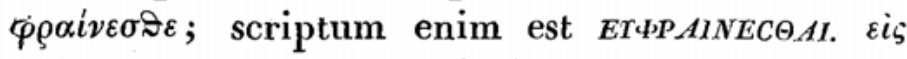

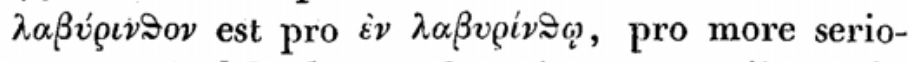




formula in epitaphiis obvia, ut Palat. VII, $69^{3}$ $\Pi o ́ \sigma \varepsilon \iota \delta o v$, ov $\sigma \dot{v} \sigma \tilde{\omega} \zeta \varepsilon$. Boschius in Anthol. T. V p. 184 Miletum, quum Romam ludos sub Imperatore Severo spectatum venisset, in delectationem vulgi labyrinthum exstruxisse reete dicit; sed qualis hic fuerit labyrinthus, cuinam delectationis generi destinatus, nec ipse dicit nec alium novi explicantem, quem ne accensitum quidem usquam aliis invenerim labyrinthis. Ex marmore illum fuisse exstructum indicat votum pro marmorariis factum. Aliquid lucis lucramur ex basi statuae eidem Mileto positae, apud Gruter. p. 33o,

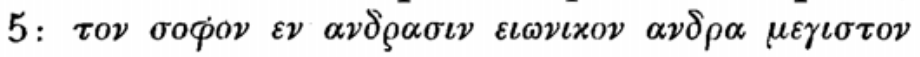

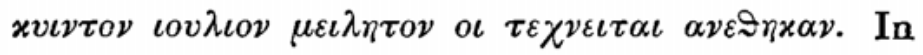

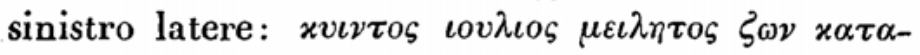
$\varepsilon \iota \varepsilon \rho \omega \sigma \alpha$ (i. e. $x \alpha \vartheta \iota \varepsilon \rho \omega \sigma \alpha) \tau \eta \pi \rho \circ \varsigma \zeta x \alpha \lambda$. $\mu \alpha \rho \tau$. Quae ad sepulcrum spectare videntur, in quo ponenda esset statua: nam sacrari eam ab eo ipso, cui ponitur, mira res foret. Perversa est Scriverii emendatio, quam Boschius adoptat, CKHNIKON ponentis pro GIWNIKon, vel, quod Gudius falso legit, OIWNIKON. Est id enim Iovıxós, et Jonicum virum artifices honorifice appellant Tripolitanum civem, quippe non in sua tantum civitate, sed in universa Jonia summum virum. Alibi 'I nomen est proprium (Corp. Inscr. n. 193 l. 19). $\mathrm{T} \varepsilon \chi \nu \tilde{\imath} \tau \alpha \iota$ saepe sunt histriones, eoque in errorem

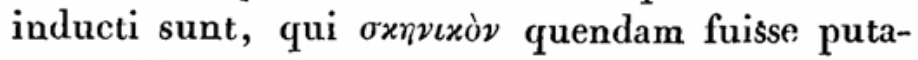
runt Miletum, hominem immensarum divitiarum 
et quae ad aedificium publicum Romae exstruenlum illa aetate, quae Septizonium surgere vidit, privato sufficerent. At alio manu ducit artificum mentio. Nonne enim ipsos, qui Mileto statuam ponunt, histriones vel mimos sive pantomimos labyrintho ad populi oblectamentum usos esse $\mathrm{di}^{-}$ camus? Certaminibus musicis Domitianus Odeum, Hadrianus Athenaeum exstruxerat. Simili aedificio nomen labyrinthi vetusta fama inclytum, et licantis vanitati plebique novitatis studiosae gratum, quo etiam Attalus, Lepidus triumvir aliique usi sunt, impositum esse videtur. Quod ad-

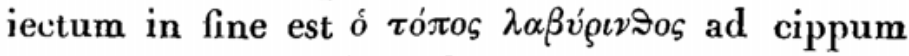
spectare puto, cui quidem locus sit labyrinthus, qui in eo vel prope illum collocandus sit. Ubinam enim aptius memoria hominis coli potuisset?

Admonet hoc epigrammatis Catanensis, cuius ego quum lacunas ita explevissem, ut fontis perpurgati et canalis ducti monumentum esset, Tu in Not. crit. p. XCVII sepulcrale esse coniecisti, ita ut cippus iuxta opus in civitatis commodum confectum, in quo ille vitae labores absolverat, quod ultimum fuerat operum eius, positus fuerit. In quo prorsus Tibi assentior: et verum Te re-

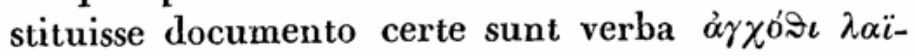


demum bene habet si antea mentio iniecta fuerit defuncti. Hoc tantum verbis non videtur inesse,

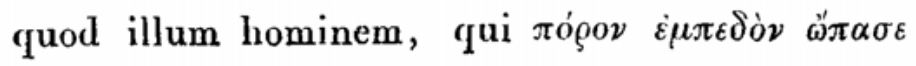





impeditum statuis canalem non perfecisse: neque diversa esse opera crediderim, de quibus Latina et Graeca inscriptio agit. Münterus in Itiner. Neapol. et Sicil. p. 427 istum lapidem supra Thermarum ostium inventum refert, et nomen versibus subscriptum ENNOIOr (i. e. Ennii) poetae esse conicit, quod verissimum puto. Etiam sub carmine in Sphingis digito insculpto poetae nomen scriptum videmus n. 17o, et Aelianus ipsis versibus in Homerum scriptis Append. n. 279 nomen suum intexuit. Amici quum interdum epitaphia componerent, ut in Megarensi fragmento ap.

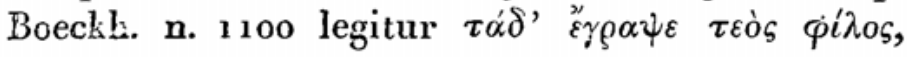
fieri vi: potuit, quin unus et alter simul nomen suum in amicitiae memoriam apponeret. Et nescio an hinc factum sit, ut bina saepe in eodem cippo insculpta sint carmina, quum plures amicitiae suae monumentum exstare vellent. Alios privatorum quoque sepulcrales titulos poetas auctores habuisse, a propinquis ad illos scribendos invitatos, quis dubitet? Euripides hunc morem tangit Troad. 1195, ubi Hecuba de Astyanacte:

Tí xaì

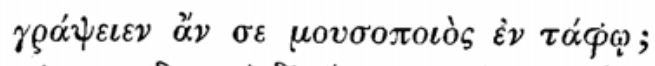

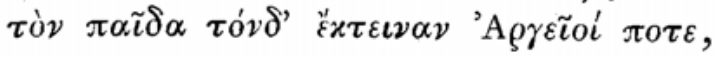

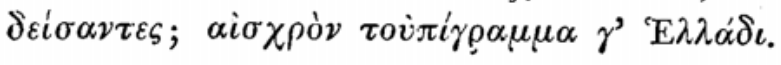

Atque horum etiam nonnullis suam una cum de- 
functi memoria praetereuntibus commendare fortasse placuit. Nomina a'xtem una cum titulis a periegetis transcripta esse coniicias, et Plutarchus Themist. 32 totis libris $\pi \varepsilon \rho \grave{i} \tau \tilde{\nu} \nu \mu \nu \eta \mu \alpha_{-}-$ $\tau \omega \nu$ a Diodoro Periegeta scriptis usus est. Ex istiusmodi libris haustae videntur inscriptiones Philiadae Megarensis in eos, qui in proelio contra Persas occubuerant, Append. n. 94, et Asinii Quadrati in inimicos gloriosa morte interemtos, Anthol. VII, $3_{12}$, quas utrasque Stephanus Byzantius servavit, fortasse etiam epitaphia privatorum a Carphyllida, Xenocrito Rhodio, Pisandro Rhodio, non vetere illo Camiraeo, Philippo Carystio (Anthol. VII, 260. 29י304. 394) scripta, quorum omnium nec nomina aliunde, neque alia carmina nota sunt praeter unum aliud Carphyllidae epigramma, et multi alii eiusdem libri septimi tituli.

In repraesentando textu eam mihi praescripsi legem, ut in is omnibus, in quibus versificator peccasse videretur, marmora exprimerem, neque contra illorim fidem vel rhythmum vel dictionem emendarem et emacularem, ut solent, qui justa carmina a poetis diligentia docta elaborata et polita edunt. Quem errorem multi olim commiserunt, et ipse Ruhnkenius, ob hoc traxatus a Marinio (Fr. arv. p. 433)*), ut in marmo-

*) Ruhnhenio etiam ap. Koen. ad Gregor. p. Jo6 titulum




ribus tractandis eadem libertate critica uterentur, qua libri veteres corriguntur, quamvis etiam non libri saepe, sed scriptores ipsi emendantur et perpoliuntur, in eum hodie non facile labaris. Ipsis poetis in epigrammatis publico usui destinatis fortasse major quam in longiore poemate licentia et in usu verborum et in rhythmo atque prosodia concessa fuit. Alii quantum licitum esse crediderint, versibus atque verbis demonstrant. Prae ceteris epitaphia multa auctores habuerunt scribendi artis rudes et ignaros. In quibus si quando boni pessimis misti inveniuntur versus, hos ex melioribus epigrammatis in ore hominum versantibus desumtos esse, membra etiam versuum et phrases novo usui accomodatas facile distingui ab iis, quae de suo homines de plebe adsuerint, Tu olim, Jacobsi, in litteris ad me datis observasti. Latina quaedam epitaphia disertam auctoris mentionem faciunt, velut Anthol. IV, 23 :

Te rogo, praeteriens, fac mora et perlege versus, Quos ego dictavi et jussi scribere quendam.

In quibus male Burmannus corrigi vult fiat mora; legendum enim mora', moram. Publius Ael. Ulpius ibid. n. 33 ipse suo curam titulo dedit, et n. 269 :

Hoc genitor cecinit Taurus lacrimabile carmen. Interdum etiam tenui hoc in genere gloriolam 
quacsitam esse, ex Luciano liquet, qui Admetum, malum poetam, epigramma monostichum ab ipso factum, quod posui n. 112, cippo suo insculpi in testamento jussisse ait, et ex Lucillii epigrammate Anthol. XI, 31 2, ubi Marcum poetam cenotaphium exstruxisse narratur, insculpto epigrammate in puerum monosticho, quod ostentandi ingenii caussa composuerat. Praecipua attentione formae videntur dignae, quae in epigrammatis occurrunt

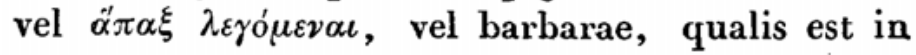

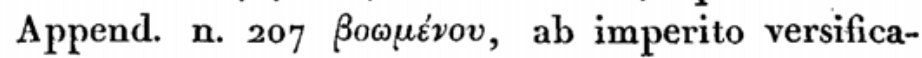
tore inventum, uti annotasti, ad supplendum versum, et quales in hac collectione sunt n. 23,

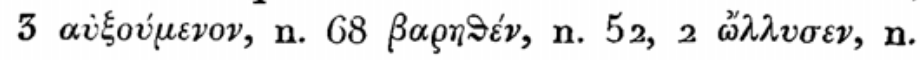

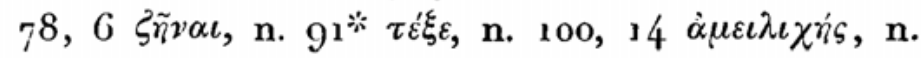
128 \%. $302 \dot{\varepsilon} \xi \eta \rho \gamma \dot{\eta} \sigma \alpha \tau o$, n. $210,5 \dot{\varepsilon} \pi i o v \sigma \iota$, et fortasse n. 136, 3 pro xєCOMAl qui scripsit non $\chi \varepsilon \dot{v}-$

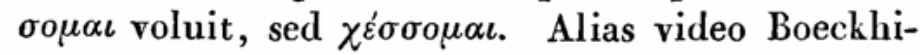
um congessisse in Corp. Inscr. T. I p. XIX et 799, qui etiam p. 12 verissime monet, dialectorum tam mirificas esse varietates, ut si forma vel maxime inexspectata clare scripta appareret, deberet ferri. Et quasdam ferendas esse etiam in antiquis scriptoribus formas pravas, recte contendit Buttmannus Gramm. ampl. T. I p. 121 not. **). Aliud soloecismorum genus est in usu particularum vel expletivarum, ut, n. $\sigma_{7}, 3 \quad \dot{\varepsilon} \nu \delta^{\prime} \alpha_{\rho} \alpha$

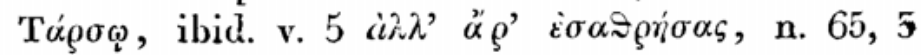

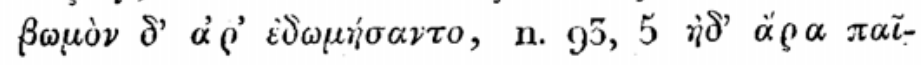




\section{- xxiv -}



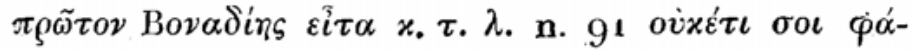

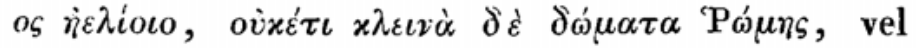
male positarum, ut $\delta \dot{\varepsilon}$ in epigrammate a. 1825 prope Ficuleam reperto:

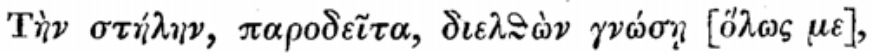

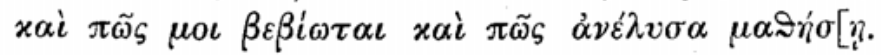

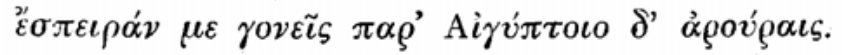

Haud melior per eandem particulam transitus fit n. 46,5 . Metri leges in multis labentis Graeciae epigrammatis violatas esse, monuisti in Praefat. Palat. p. XVII not. 11. Maximam in versibus incuriam prodit pedum numerus non justus, uno pede vel deficiente, ut hexametris n. $37,2.46,3$. 53 , 1 , vel ultra mensuram addito, ut et pro senariis versus habemus septem pedum n. 59, 4. 134, 10 et 11, qualem continet etiam Anthol. Lat. IV, 323 , 2, et pro hexametris, $\Lambda$ ppend. n. 315, 5, ubi nomen et numerum annorum non cepit versus integer, Syllog. n. 6. 34, 1 ; et hi quidem quum versus boni sint et a multis fortasse antea usurpati, serius interpolati esse videntur, ut de alio Tibi Not. crit. p. XCV v. ? visum est et Boeckhio etiam n. 1064. Spondeus in quartam trimetri sedem irrepsit n. 23, 2. De aliis trimetris n. 196 non loquor. Hiatuum in versibus serioris aevi exempla ex Anthologia protulisti in Praefatione p. XXXVIII not. 38, nec in hac minore Sylloge 
eorum est penuria. Legitur n. 93, $7 \mu \dot{\varepsilon} \dot{\varepsilon} \delta \varepsilon i \mu \alpha \tau o$,



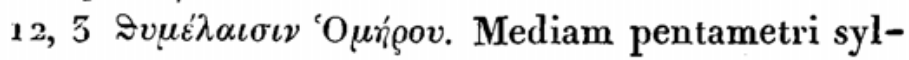
labam in caesura brevem habemus n. 82, 4. 88, 4, quibus augeatur Friedemanni catalogus p. 284, qui Lucianum primum post artis excultae tempora sibi hanc indulsisse libertatem statuit. Eadem usus est Ennoeus in epigrammate Catanensi v. 6. Contractiones duriores notavi ad n. 11/. In

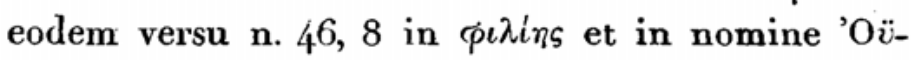

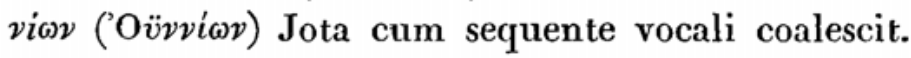
Incredibili licentia multi usi sunt in syllabarum productione et correptione, quarum exempla lu-

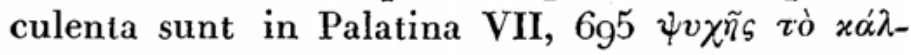

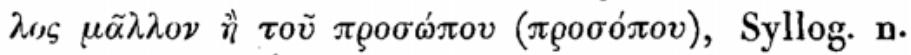


prima longa, in trimetro. In thesi passim breves syllabae producuntur, ut n. 11, $6 \pi \tilde{\alpha} \sigma \iota \nu \grave{\alpha} \gamma \gamma \varepsilon \lambda \dot{\varepsilon}-$

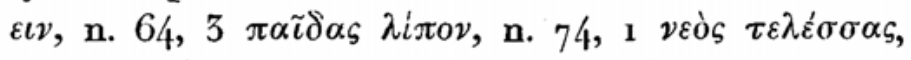
n. 100,15 $่ \varsigma$ จ่์ov, cf. Animadv. ad Anthol. T. II P. 2 p. 30o, et in arsi etiam producitur $\pi \alpha \tau i \eta \rho$

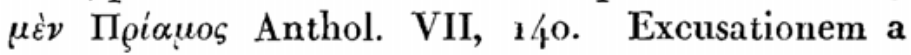

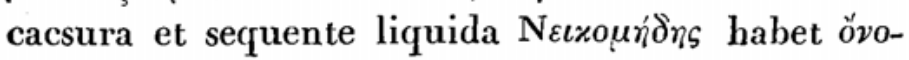

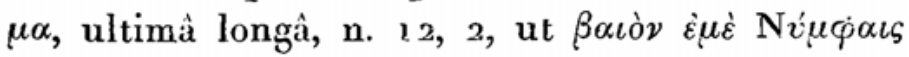
in epigr. Catanensi, $\alpha \dot{\alpha} \mu \alpha$ Nv́ $\mu \varphi \alpha \iota \varsigma$ Odyss. VI, 101,

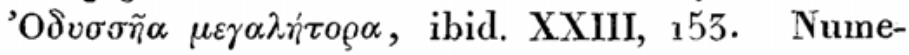


terat n. 38, 2. Socordiae vel inscitiae tribuas $n$. $83,3 \delta \varepsilon \iota \sigma \iota \delta \alpha i \mu \omega \nu$, prima correpta, n. $9^{3}, 4 \dot{\alpha} \psi i$ - 
$\delta \alpha$, ut $x \nu \alpha u \iota \delta \varepsilon s$ ap. Alcaeum, Athen. XIV p. 637 B, $x \rho \eta \pi i \delta \alpha$ ap. Pind. monente illic Gu. Dindor-

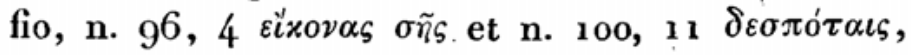

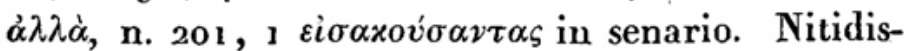
simus hexameter est n. $9^{3}, 6$, comparandus cum disticho Append. n. 233, 3. 4. Nomina propria cum

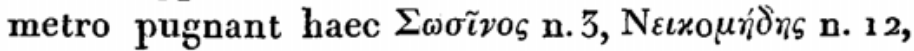

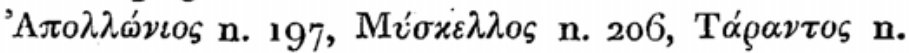





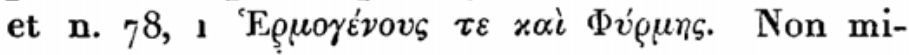
nor fit vel auri vel litteris vis Append. n. 28 in $\Pi \alpha \sigma \iota \sigma \omega x \lambda \tilde{\eta}_{\varsigma}$, n. 47 in 'A $A \varepsilon^{\prime} \xi \alpha \nu \delta \rho \circ \varsigma$, n. 106 in $\mathrm{M} \alpha-$

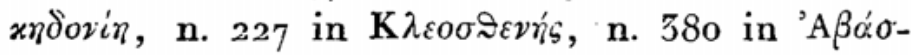
$x \alpha \nu \tau \iota \varsigma$, quod nomen obvium est in Atticis titulis, Smyrnensibus, Nubiensibus et Latinis, n. 402 in $\mathrm{B} \alpha \sigma i \lambda \lambda \eta$, Not. crit. p. XCIII in viòs $\Phi \iota \lambda i \pi \pi \sigma v$. Exempla correptae in nominibus vocalis $\bar{\omega}$ sunt allata in Animadv. ad Anthol. T. ult. p. 826, productae litterae $\bar{\imath}$ et $\bar{\alpha}$ in is, quae in $\iota v o s$ et $\alpha \nu \circ s$ exeunt, ibid. p. 13 et $7^{6}$ et Not. crit. ad Palat. VII, 7oo. IX, 510, Append. n. 247, 15. $271,1$. 381, 1. Sic Juliänus Anthol. Lat. IV, 226.

Lectionem epigrammatum etiamsi vitiosa sit marmorum fidei astringendam dixi. Non verendum autem est, hac via ne conjecturae et correctioni materies praeripiatur et ingenii critici exercendi parum copiae relinquatur, quod potius nescio an vel sic in nullo quoquam scriptorum 
genere plus pabuli inveniat, quam in epigrammatis. Scatent enim vitiis cum quadratariorum serioris temporis marmora plurima, tum transcribentium schedae et textus editi multi lapidum cujusque aevi. Litterae et syllabae omissae sunt, perperam geminatae, confusae, saepe singuli versus explendi et reficiendi, ipsa verba ex litteris continuis formanda haud raro ambigua sunt, totum saepe argumentum divinatione critica constituendum et rationibus suis fulciendum. Et de vitiis orthographicis et ceteris, quae non versificatorum culpa, sed lapicidarum et transcribentium inscitia et oscitantia orta sunt, velles saepe, his an illis tribuenda sint, constaret; nimium enim parvus numerus est apographorum, quorum fides omnino certa sit. In nostra quidem Sylloge, quamvis in plurimis titulis suspicionis caussa non adsit, pro certo tamen eorum tantum aflirmare ausim veram ubique scripturam vel editam vel in annotatione exhibitam esse, quos Romae Gerhardus transcripsit et quos in Parisino Museo ipse examinavi. In his igitur quaecunque vitia commissa sunt, lapidariis debentur, ut n. 46, 2 HMAPTON pro $\hat{\eta} \mu \alpha \rho \tau \tilde{\omega} \nu$, si vera est conjectura, v. 6 OXNOMA pro erMIMA, n. 55, 10 NHwI pro NHCOI, n. 57,2 METEMONTECOP.AC pro $\mu \varepsilon \tau \varepsilon^{\prime} \chi O \nu \tau \alpha$, et quae praetera sensum pervertunt n. 6o, 6. 61, 2. 68,1 . In his igitur locis correctione opus est. Sed ultra progrediendum, nec videndum tantum, 
quid scripserint versificatores, sed saepe etiam, id, quod Tuarum emendationum elegantissimarum plures praestant, quomodo scribere debuissent. Ceterum etiam in tanto Anthologiae locorum depravatorum necdum 'certis conjecturis sanatorum numero superesse nonnullos puto, in quibus emendatione abstinendum sit, quia ex scripturae ductibus verus sensus elici possit. Quorum unus est, de cujus explicatione spero et confido Te mihi assensurum esse, doctissime Jacobsi, in eleganti epigrammate Erinnae, non Lesbiae illius, quod in Catalogo poetarum epigrammaticorum monuisti, sed ignotae poetriae, Palat. VII, 712, quod quam varie tentatum sit ad Anal. T. I p. 58 n. 3 et in Add. p. $39^{2}$ exposuisti. In hoc igitur lectionem tueor Codicis ab omnibus rejectam $\tau \alpha$ $\mu \varepsilon \vartheta^{\prime} \dot{o} \rho \tilde{\omega} \nu \tau \iota$ ơ $\mu \mu \alpha \tau \alpha$, scribens $\tau \alpha^{\prime} \mu \varepsilon \approx 0 \rho \tilde{\omega} \nu \tau \iota$, Dorice ut $\dot{\alpha} \gamma \gamma \varepsilon \lambda \varepsilon \dot{\varepsilon} \sigma \tau$ : in v. sq. Vocalis enim in $\tau \dot{\alpha}$ producitur ante liquidam ut apud Callimachum

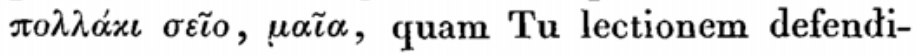
sti Not. Crit. p. 1043, et saepissime apud Homerum ut in $\Delta i \hat{\imath} \mu \tilde{\eta} \tau \iota \nu, \hat{\eta} \delta \dot{\varepsilon} \mu \dot{\varepsilon} \gamma \alpha \nu$ i $\sigma \tau \dot{\nu} \nu$ Il. III, 125, $\tau \varepsilon \mu \varepsilon \gamma \alpha \dot{\lambda} \lambda \eta \nu$ ib. 221, $\delta \varepsilon \tilde{v} \rho \circ \mu_{\varepsilon} \nu$ ibid. $240, A i-$

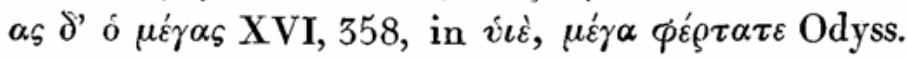

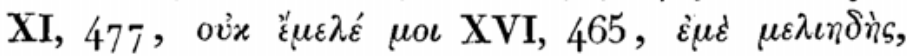

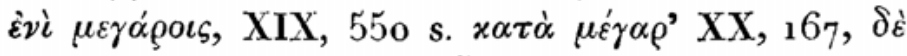

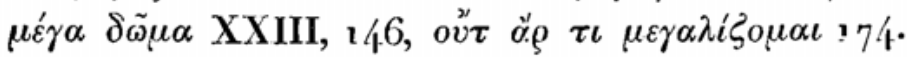

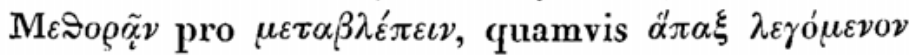
est, qualia multa sunt in epigrammatis, non ha- 
bet difficultatem. Atque his admissis verbis verum demum ajo prodire carminis argumentum, novum id, gratum atque venustum. Quod Salmasio, Brunckio atque Tibi placuit, mi Jacobsi, figuris allegoricis monumentum ornatum fuisse ( $\sigma v \mu \beta o \lambda \alpha$ vocat Antipater Sid. Epitymb. n. 425, 10), quibus sepultae mulieris mors ipsas consecuta nuptias significaretur, unde ó $\mu \mu \alpha \tau \alpha \tau \dot{\alpha}$ mutandum fuit in ஸ่ $\mu o \tau \alpha \dot{\tau} \alpha \nu$, Tu leniore atque feliciore, quam ceteri, emendatione ita expressisti :


$\delta \varrho \tilde{\nu} \tau \iota$

$\grave{\omega} \mu \circ \tau \alpha^{\prime} \tau \alpha \nu$ B $\alpha v \varkappa o \tilde{v} s \dot{\alpha} \gamma \gamma \varepsilon \lambda \varepsilon \dot{\varepsilon} \sigma \tau \iota \tau v^{\prime} \chi \alpha \nu$.

Et in Palatinae quidem editione dubitas, $\sigma \alpha-$ $\mu \alpha \tau \alpha$ de inscriptione ipsa accipiendum sit, an de signis quibusdam symbolicis cippo insculptis. De illa si cogitemus, nescio an affectati quid habeat epitheton $x \alpha \lambda \alpha^{\prime}$; haec si praeferas, non video, quibusnam symbolis significari potuerit, Baucidem, non dico ipso nuptiarum die, quod omnino symbolico modo exprimi nequit, sed nubilem et aetate florente mortuam esse, nisi sculptus fuit Pluto sponsam rapiens, qua imagine nulla fere frequen- tior fuit in sarcophagis et cippis. Hanc ipsam autem fabulam indicant verba $x \alpha \lambda \dot{\alpha} \tau \dot{\alpha} \mu \varepsilon \approx 0 \rho \tilde{\nu} \nu \tau \iota$ óm $\mu \alpha \tau \alpha$, quod ut ostendam, totum carmen transcribo, ita quidem ut v. $4 \tau \dot{\alpha}$ et $\tau v^{\prime} \chi \alpha \iota$ cum Salmasio et Reiskio mutem in $\tau \dot{\alpha} \nu \tau v^{\prime} \chi \alpha \nu$, et in di- 
sticho tertio Tecum suppleam $x \alpha \lambda \dot{s} \varsigma$, atque $\dot{\omega} s$ ponam pro $\hat{c} \varsigma$ et $\tau \alpha \tilde{\imath} \sigma \delta '$ pro $\tau \dot{\alpha} \nu \delta^{\prime}$, praeterea autem v. 5 pro $\tau \dot{\alpha} \delta \dot{\delta}$ scribam $\tau \alpha \dot{\delta} \delta$.

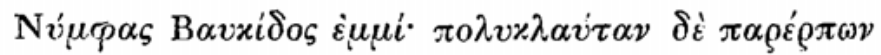

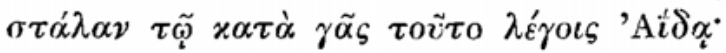

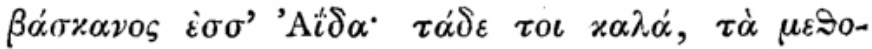
$\rho \tilde{\nu} \nu \tau \iota$,

ô $\mu \mu \alpha \tau \alpha \tau \dot{\alpha} \nu \mathrm{B} \alpha v \times o \tilde{v} \varsigma$ $\dot{\gamma} \gamma \varepsilon \lambda \dot{\varepsilon} \sigma \nu \tau \iota \tau v^{\prime} \chi \alpha \nu$,

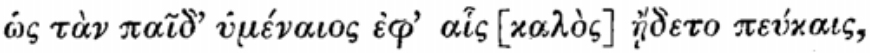

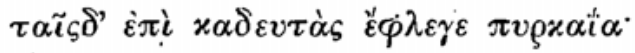

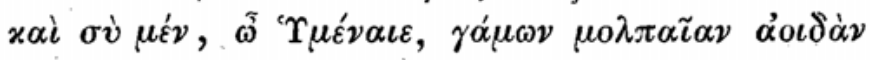

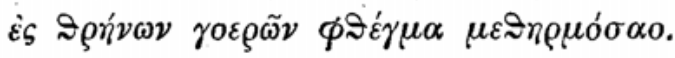

In anaglypho toties repetito, ad quod hos refero versus, nihil est significantius, nihil etiam magis constanter expressum, quam caput sponsae a raptore aversum et retortum, vultus ad vitae amoenitatem retrospiciens, moeroris et desperationis index. Hic igitur vultus loquitur quae sors fuerit Baucidis, $\tau \alpha^{\prime} \delta \varepsilon \varkappa \alpha \lambda \dot{\alpha}$ ŏ $\mu \mu \alpha \tau \alpha, \tau \dot{\alpha} \mu \varepsilon$ o $\rho \tilde{\omega} \nu \tau \iota$, vultus, non aspicientis, puto, quamvis nec hoc ineptum, sed respicientis: nam aspicere licet vulgo significet $\mu \varepsilon \tau \alpha \beta \lambda \varepsilon^{\prime} \pi \varepsilon \iota \nu$, quidni praepositio in hoc composito etiam eam habere possit vim, quae est in $\mu \varepsilon \tau \alpha \sigma \tau \rho \varepsilon \dot{\varepsilon} \varphi \varepsilon \sigma \nabla \alpha \iota, \mu \varepsilon \tau \alpha x \lambda i \nu \varepsilon \iota \nu, \mu \varepsilon \tau \alpha \dot{\rho} \rho \varepsilon \tilde{\varepsilon} \nu$ ? Ceterum

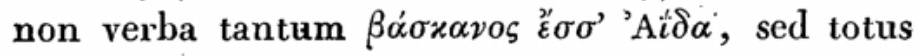
sermo viatoris est; quum enim in primo disticho cippus loquatur, tum verba sequantur viatoris, non potest denuo mutari persona et ad poetam 


\section{$-\quad \mathrm{XXXI}-$}

transire oratio. Cippus autem viatorem monet, ut et Plutonem et Hymenaeum alloquatur, quae ingrata foret repetitio, nisi oratio ad alteram personam in marmore repraesentatam converteretur. Hymenaeus est puer alatus cum face Plutonis currui velut dux praevolans, vario nomine Amor etiam et Hesperus vocatus, de quo dixi in Diario Gottingae edito de arte ant. p. 83. Quo spectat etiam Meleager in ep. 125 in Clearistam (Palat, VII, 182):

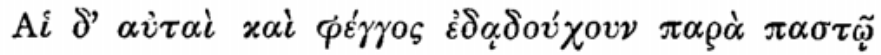

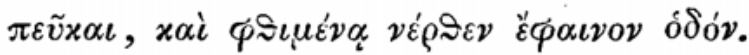

Atque sic demum v. 2 bene habere videtur. Mirum enim foret si viator Plutoni, ad quem quidem iter non dirigeret, et ante quam de illius crudelitate et de sepultae mulieris fato aliquid accepisset, quaedam dicere iuberetur. Contra si Plutonem raptorem supra vel infra verba sculptum tibi finxeris, nihil desiderabis. Res ipsa imagine indicata est, additur inscriptio quasi explicandi caussa et ad pingendum, quem illa moveat, affectum. In simili carmine Epitymb. n. 186, 5 Antipater Thessalonicensis :

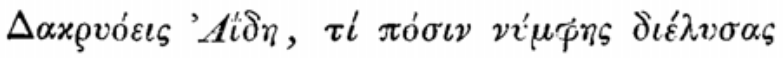

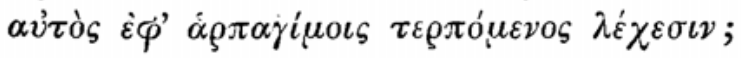

Verbo meam de hoc epigrammate sententiam indicaveram in libro modo citato p. $19^{6}$, ubi etiam 
p. $9^{1} \cdot 19^{5}$ cippi Romani quinque recensentur eadem ornati imagine, quam nec in eo desse suspicor; unde Maffejus in Mus. Veron. p. LXI epitaphium edidit in Liciniam Pollam, in Append. n. 229. Potest tamen epigramma nostrum, nisi epideicticum fuit, id quod duplex nominis forma Bavrò et Bavxis prodere videtur, etiam sarcophagus insculptum habuisse; nam sarcophagos frequenter epitaphiis instructos fuisse vel hic libellus docet n. 3 ₹. 56. 66. 75. Et in eo, de quo p. $29^{G}$ dixi epigramma ad ipsas, quibus sarcophagus ornatus est, imagines spectat. Carmen Erinnae in Baucidem imitatur epitaphium vel Leonidae vel Meleagri in Erinnam Lesbiam Palat. VII, 13, quod in Epigrammatum Delectu posuisti IV, 33:

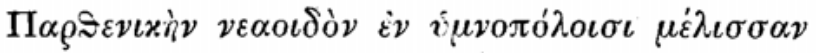

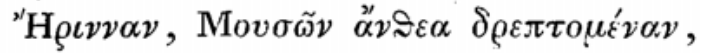

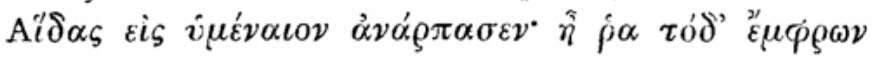



Quae adiunxi, ut hunc quoque poetam ad aliam eorundem in sarcophagis anaglyphorum partem spectare monerem. Solet enim in plurimis Proserpina flores in pratis Ennensibus cum Venere, Pallade et Diana decerpens a Plutone occupari. Cum his gitur floribus carmina comparantur, quibus intenta poetria a Plutone rapitur.

In enotanda lectionis varietate non eandem in omnibus titulis rationem secutus sum. Qui 
enim in Corpore Inscriptionium iam sunt editi, in quo quasi horreo vasto et verae lectiones et falsae maximo acumine maximacue cura selectae et. congestae ad seram posteritatem asservantur, eorum non nisi eas annotavi scripturae diversitates, ex quibus de eiusdem vel emendationis veritate iudicari et de quibus adhac disceptari possit; in ceteris quicquid inveni varietatis apposui, simulque editorum notas, quae ad rem facere viderentur, excerpsi, curavique, ut libri minus vulgares, ut Acta Academiae Gallicae et Petropolitanae, Itineraria multique alii, quibus usus sum, ii ut lectori curiosiori omnino ne desiderandi essent. Et quae ad linguae et ad argumenti rationes pertineant, ea etiam ex Corpore Inscr. adiunxi, omissis tantum ex Boeckhiana annotatione iis, quae non ad eruendum verum, sed ad refellendas falsas opiniones scripta sunt; atque ita etiam de meis rescidi, quae hoc consilio in priore editione de quibusdam titulis, nunc ab amico uberrime tractatis, animadversa erant. $\Lambda d$ varietatem lectionis tamen non arbitror pertinere quidquid in scripturae ratione variatum

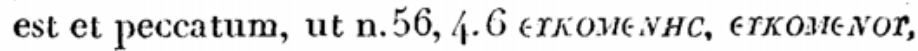
quae mutatio admodum frequens (Grut. p. 1137

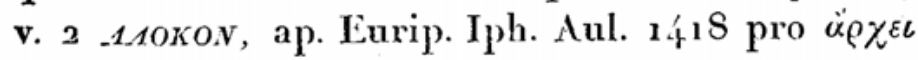
emendatum est $\dot{\alpha}(\hat{x} \varepsilon \tilde{i})$, neque omnia, quae veteribus propria ab hodierno usu recedunt: Non notavi igitur legi EVENK-AMENHN n. 10; 4, ENTE.AA$\Sigma A \Sigma$ n. 12, 4, ANANKH n. 13, 3 et 184, 12, .1.ANKIOr 
n. 55, 3, $\in N \Gamma T C$ n. 100, 5, ut ap. Boeckh. n. 22, et similia passim: $\vec{v}$ autem pro $\mu$, IENIIOI $\Sigma$ n. 36 ,

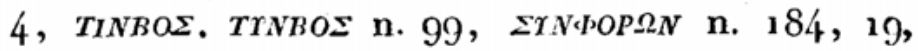
AMENILES in Naniana n. 119,1 , ut EXNMAXIA, o.1rNHIOI in Elea, et $\bar{\mu}$ pro $\bar{v}$, ALA n. 18 , ГНРАІОМ ВIOTАI n. 45, 10 , K.AM IEIMENOI-

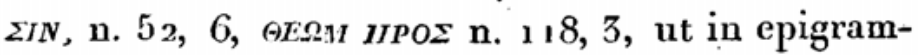
mate Megarensi ap. Boeckh. n. 170, 1 ERIO.AEMOM [MNHMIA], MEM

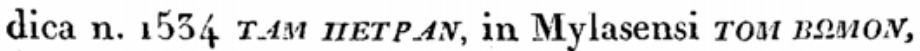
et ut hodie quoque Graeci scribunt $\tau \dot{\eta} \mu$ สó $\mu \pi \eta v$. Neque Jota subscriptum vel positum vel omissum commemoravi; quamvis vellem nunc positum, quod rarius in his titulis, semper indicassem. In Boeotia maturius' illud omitti coeptum est, v. Boeckh. in Corp. Inscr. T. I p. 783 . 700. 80. Circa Olymp. 145 jam deesse in p.Ains et similibus formis, non tamen constanter, idem annotat p. 770 . Notissimus est locus Strabonis XIV p. 648: $\pi \circ \lambda \lambda$ oi

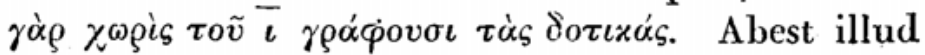
a monumentis Aphrodiensi et Stratonicensi ad triumvirorum Romanorum aetatem pertinentibus ap. Chishull. p. 152. 156, notante Koenio ad Grcgor. p. 607 , etsi monumenta publica diutius quam privali et in libris et in lapidibus adscribendi illius morem conservarunt. Quintilianus I, 7, 7 Graecos ¿ litteram non solum dativis casibus in parte ultima adscribere (sua igitur aetate), sed quibusclam etiam interponere ait. Imperatoris Com- 
modi aetate certarunt Grammatici, $\varepsilon l \tau \alpha \tilde{\iota} \varsigma \delta \delta \tau \iota-$

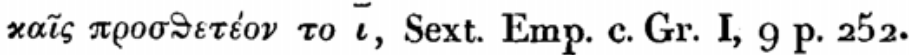
Non deest Jota in eximio epigrammate Philano, Augusto imperante inciso n. 169, 2. 3. 10; adscriptum est etiam ubique in oraculo Pergameno n. 183, quum desit semper in Patarensi n. 184; et illud quoque serioris aetatis esse prodit $\bar{\varepsilon} \iota$ pro i scriptum. In epitaphio Thebano n. 26 legitur $\mathrm{x} \Omega P A I$ et nOAEM $\Omega$, in monumento Amazaspi lberi, cujus Romae fuisse videtur sepulcrum honora-

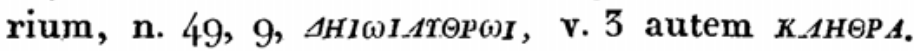
Epigramma Corcyraeum n. 32, 6 habet $A\left[\left[\Sigma_{E N}\right.\right.$, v. $14 . A K M A I$ et $\Omega 1$, v. 3 autem T.AWH $\Sigma I$ et K-AHZETAI, v. 16 rnA $1 \Omega$ : Aliquoties etiam in scrioribus titulis appositum reperitur Jota, ut in eo, quem p. 46 ex Walpolio transcripsi, in alio Walpoliano Travels p. 589 ex imperatoris Severi tempore, n. $40,3 \omega 1$ et in eodem versu $\epsilon A r T \omega$, n. 79,6

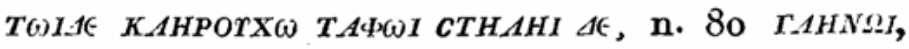

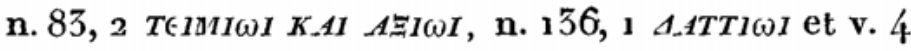

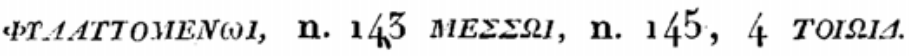
Praeterea ubique, si bene attendi, omissum est, notavi certe ad n. 8,3 et 4. n. 29, 2. 34, 1. 35, 4. $41,6.42$, 4. $46,1.53,2.3 .5 .55,9.13 .15$. 57, 2. 3. 4. $61,1.62,3.68,2.121,6.134,10$. 137 , 2. $150,3.159$, 4. In Nubiensibus inscriptionibus Niebuhrius semel, tab. IV, 23, additum invenit; semper deest etiam in Syriacis; in Memnonianis et in $\pi \rho \sigma \sigma x v \eta \mu_{\mu \alpha \sigma}$, praeter unum ap. Wal- 


\section{- Xxxvi -}

pol. Travels p. 591. Minime omnium opus videtur, ut quoties elisionem neglexerint lapidarii in editione non primitiva annotetur. Non magis enim quam librarii hac in re sibi constant illi, et saepe in eodem lapide admodum variant, v. ad n. $183,10$. Exempla plura collegi ad n. 32, 16. In pluribus antiquissimorum abjicitur vocalis in hiatu et in crasi, ut n. 117 OOEPMEZ, n. 119 TOLAMENIIHES, TOTTETEAE YY E, n. 122 T]-AFTTOY, n. 123 T-APTEIOI, n. 124 TTP-AN.AIIO, ap. Boeckh. n. 22 TO.JEF STEEEN (in columna striata n. 120 est TO.AE.1CA.1ILAIEPESI $\Omega$ A $[\sigma \iota v]$, et ita interdum in serioribus apostropho usi sunt, ut n. 54, 6 KEIN, n. 60,4 KOT: sed multo frequentius posuerunt diphthongum in crasi v. ad n. 52,4 p. 76 , et vocalem in hiatu abjiciendum n. $6,2.13,2.15,4.26,4.45$, 4. 7. 10. $46,1.2 .5 .59,1.3 .6 .60,2.61,5.73$, 3. $79,1.100,5.5 .11 .15 .102,5$. 4. 1466 1. 150, 1. 3. 158 , 1. et 5 (TE.19.10\%OPOI:), 164 , 1. n. 55 , 4, quo in titulo praeter $T E$ HNOPEHN hiatus in scriptura non admissus est.

Legitimum verborum Graecorum sonum posteriorem aetatem latuisse, et plus etiam incuriae et ar:sitrio tribuendum, verissime monent Animadv. in Anthol, T. XII p. $31_{2}$. Sunt tamen in ipsis mendis scripturae leges quaedan! analogiae, quas nisi observaverit et stabiliverit, multum operae et temporis perdet Criticus, et ensendationes multas frustra excogitabit. Quare vitia manifesta. 
aliquoties commemoravi et contuli, et alia fortasse addere debueram, ut geminum $\Lambda 1$ pro $M$, quod inveni n. 195, ] П૯PIKAMEA, n. 197, 1 AHOM $\Omega N I-$ $o \Sigma$ et saepius in Gavii schedis. Idem vitium est in titulo Memnoniano ap. Leich. Carm. sep. p. 79 et in Descriptione Aegypti p. 111 n. 19 тРЕВОМH, bis in Ennoei epigrammate Catanensi, $A M$ pro $\dot{\alpha} \lambda \lambda \lambda^{\prime}, \operatorname{поM[-A}$ pro $\pi о \lambda \lambda \alpha^{\prime}$, ap. Dodw. Itiner. T. II p. 283 IIANEMHN.A, in titulo Siculo ap. Castell. p. 168, 27 K_AMIITP_ATOE; ap. Murat. p. 1321, 10 AMA pro 1.1.1. In iisdem litteris ap. Boeckh. n. 666

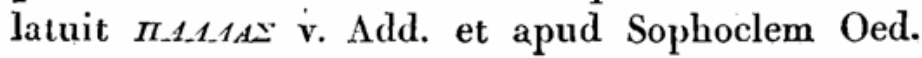
R. 530 variat in Codd. lectio $\tau \alpha^{\prime} \mu \alpha$ et $\tau \alpha^{\prime \prime} \lambda \lambda \alpha$. Hinc Callistrato c. 14 certissime restituisti ov̉x $\dot{\alpha}-$

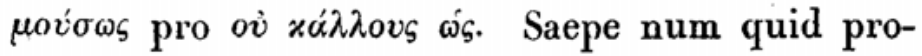
nunciationis vitio, an calami vel scalpri lapsu aliter quam vulgo scriptum sit, anceps est judicium, ut in consonantibus geminis et in vocalikus $\overline{\varepsilon \iota}$ et $\iota$ confusis. Ad consonantes quod attinet, sphalma apertum est n. $59,9 \Sigma T H_{\perp 1} 1 H$, n. $19^{3}-1 \times 1 \_1.1 H I$; in vase in Millingenii Monum. ined. tab, $38 \mathrm{HE}-$ P_AK_1_1E: Nomen unius ex Oenomai equabus ap. Scholiast. Apollon. I, 752 et Phavorinum $\Psi i \lambda \lambda \alpha, \Psi v i \lambda$ $\lambda \alpha$ est quum Bacchi cognomen Laconicum rectius $\Psi i \lambda \alpha \xi$ a Pausania scribatur; a $\pi \tau i \lambda o v$ enim utrumque descendit. Saepe autem liquidae conduplicatio in scriptura ipsam pronunciationem imitari videtur $u t$ in iis, quae ad verbum AHMMA $\mathbf{n}$. I61 p. 294 congessi, et in ipso fortasse verbo $\lambda \tilde{n} \mu \alpha$. 


\section{- Xxxvir -}

Hoc etiam cadere videtur in duplicatum sigma inter vocales duas et ante ' $T$ et $\mathrm{K}, \mathrm{X}$. Scribitur enim


Terpandrum Lydi de Mens. c. 5 p. 81 , Stephanum,

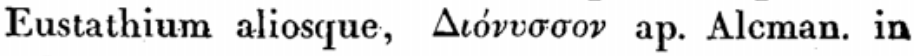

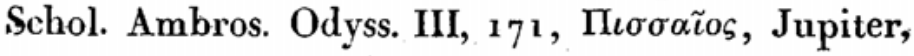

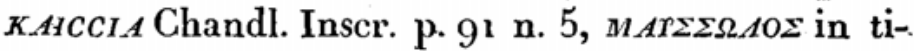
tulo Mylasensi ap. Leakium Itiner. As. Min. oro-10rcEIANOC in numa Musei Vindobon. et Quinctilianus I, 7, 20 Ciceronis temporibus paulumque intra, fere quoties $S$ littera media vocalium longarum vel subjecta longis esset, geminatam refert. $U$ t caussae, cassus, divissiones quomodo et ipsum et Virgilium quoque scripsisse, manus eorum doceant. Gemini ante consonantem sibili exempla plurima attulit Boeckh. ad n. 25. 429. 1306. 1520. 1638 et occurrunt alia, APISETSNIAAS, arti-

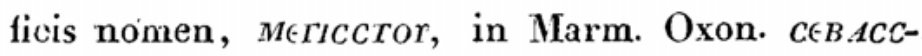
TION et ФPICCK $\omega$ in titulo Naniano, Paciaud. Mon. Pelop. T. I p. 198, $\epsilon$ CcTIN in Ephesino titulo, Diar. Class. T. IV p. 456, АCСK $A$ HILAJHC in titulo sepulcrali Romano ap. Marin. Fr. arv. p. 493,

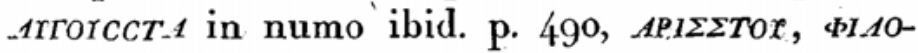
C $\in$ B.ACCTOrC in marmore Corcyraeo et aliis ibid. Mirum est de hac scribendi ratione Rosii judici: cium Inscr. vet. p. XIVI. Mihi veteres inter sibilum durum et mollem sive tenuem distinxisse videntur, fere ut Gallica lingua, in qua quod in marmore scriptum est SS.ATVRNI habet quo se de- 
fendat. Et fortior fortasse revera fuit etiam rhota cismus in XAIPPONEA, Boeckh. n. $6_{79}$, et in Kr pIOE apud eundem, pariterque sonus aliarum liquidarum, ut in $1 L_{1} A A O N$, Boechh. p. $9^{11}$, in $\Sigma A P A A-$

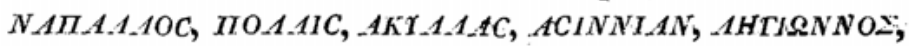
quae Marinius p. $49^{\circ}$ habet, quamvis etiam -10rחIIO $\mathrm{ab}$ eodem et Latina multa p. 500 afferuntur, in quibus consonans gemina est per mendum. In numo apud Mionnetum T. II p. 13 exaratum cst. -1.4IIIIEss. Contra non detrahitur sono, sed pro mendo vel pro scribendi compendio habendum si semel ponuntur consonantes ut IIAPHEIA Boeckh. n. $1603, K 1.11=2 T] \Omega$ n. 429 Id. cf. Add. p. 912 et alia in Elea et Sigea et quae p. $174 \mathrm{me-}$ moravi; et Knightius Proleg. in Hom. \$. 173 antiquos brevitati indulgentes tantum non ubique singulas litteras pro geminis posuisse ait. In simplici $I$ ponendo pro diphthongo EI quum in hoc sono proferendo temporum et locorum rationem habere omnino non consueverimus, etiam in epigrammatis contra lapides mori nostro est obsequendum. Scribitur autem per EI non longum tantum $I$, sed interdum etiam breve, ut $n$. 54,36 IIESEI IHCO, n. 161, I HOZEIAWNIE, n. 163, 4 ЄXORCEIN et EISNIKOC in titulo, quem supra explicavi cf. Add. ad n. 125. Constans est EI in hac nominum terminatione B.A.ANTEINH, IAR.AGI-

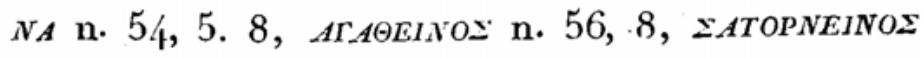

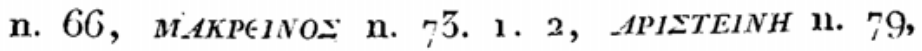

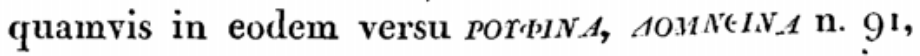
KECTEINOC n. 93; $A N T \omega I \in I N O P$ n. 200 , et omnino 


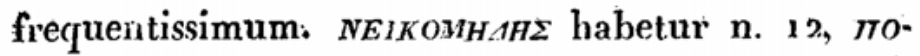
AEJTAIC n. 30. 3, EIMEPTHN, GHIEPOECC.A n. 30, 5.


TA n. $34,1.47$, 1. 51, 1. 52, 2, TEIMOQEOS, n. 48, TGIMIWI n. 85, 2, APPOAEIIIOS n. 51, HMGIN n. 52, 6, TPEINAKPIH n. 53, 1, PEIKNON n. 54, 15, KPGIN.AC n. 77, 2, AHOTEISEI n. 188, 2, BOTCGIPIN n. 190, 15, $\epsilon I C I N$ n. 196, 2. Contra $\iota$ usurpari pro $\bar{\varepsilon} \iota$ solitum esse, quod Boeotorum antiquitus fuit et Spartanorum etiam, Triopeiarum inscriptionmm tempore, in quibus $\bar{\imath}$ mutatur $\operatorname{cum} \bar{\varepsilon}$ et $\bar{\varepsilon} \iota \operatorname{cum} \bar{\imath}$, numi ostendunt, notante Viscontio ad Triop. I, 4 et 15 . Et hujus quoque usus multa epigrammata nostra continent exempla. In Bosporana $n$. 48 est TPIS atque una OIKTEIPS, in Romanis $\mathbf{n}$. 56, 2 MIOTEPHN ana cum ATAAEINOS, n. 59, 2, AIII et v. $10 s c$, n. 68 ePIchc, n. 102, 2 1.1EO\&; in Syracusana n. 163, 2 IAPOE, n. 165 AIKHAON, in Patarensi n. 184. 1. 2. 17. 19 IIP.AEIC, EEIC, AIAEIC et in eadem NEIKHAOPOE, in Syriacis n. $9^{5}$, I $\in A T$ MATO, n. $98,4 \in X I N$ et $\mathbb{L}_{\in} \in \mathrm{PIN}_{\mathrm{N}}$ in aliis n. 100,14 ic, n. 1,4, 3 AILEKTIN_1s. Ita apud Boeckh. in Atticis n. 995 et 1656, 10 AIMNHSTOE, n. 1012, l

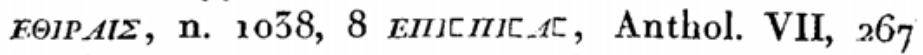

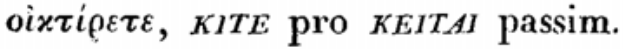

Vale, Vir optime et dilectissime, meque, ut facis, amare perge. Bonnae m. Jun. 1828. 